\title{
Drug-loaded nano-microcapsules delivery system mediated by ultrasound-targeted microbubble destruction: A promising therapy method (Review)
}

\author{
JING MA ${ }^{1,2}$, LIAN FANG DU ${ }^{1}$, MING CHEN $^{2}$, HANG HUI WANG ${ }^{1}$, LING XI XING ${ }^{1}$, \\ LI FANG JING ${ }^{1}$ and YUN HUA LI ${ }^{1}$ \\ ${ }^{1}$ Department of Ultrasound, Shanghai First People's Hospital Affiliated to Shanghai Jiaotong \\ University School of Medicine, Shanghai 200080; ${ }^{2}$ Department of Cardiovascular Ultrasound, \\ Shanghai East Hospital Affiliated to Tongji University, Shanghai 200120, P.R. China
}

Received January 14, 2013; Accepted April 15, 2013

DOI: $10.3892 /$ br.2013.110

\begin{abstract}
The nano-microcapsules drug delivery system is currently a promising method for the treatment of many types of diseases, particularly tumors. However, the drug delivery efficiency does not reach a satisfactory level to meet treatment demands. Therefore, the effectiveness of delivery needs to be improved. Based on the alterations in the structure and modification of nano-microcapsules, ultrasound-targeted microbubble destruction (UTMD), a safe physical targeted method, may increase tissue penetration and cell membrane permeability, aiding the drug-loaded nano-microcapsules ingress the interior of targeted tissues and cells. The effectiveness and exact mechanism of action of the drug-loaded nano-microcapsules delivery system mediated by UTMD have yet to be fully elucidated. In this study, the latest advancement in UTMD-mediated drug loaded nano-microcapsules system technology was reviewed and the hindrances of UTMD-mediated drug delivery were assessed, in combination with a prospective study. The findings suggested that the drug delivery efficiency of nano-microcapsules mediated by UTMD was distinctly improved. Thus, the UTMD-mediated drug-loaded nano-microcapsules delivery system may significantly improve the efficiency of drug delivery, which may be a promising new therapeutic method.
\end{abstract}

Correspondence to: Professor Lian Fang Du, Department of Ultrasound, Shanghai First People's Hospital Affiliated to Shanghai Jiaotong University School of Medicine, 100 Haining Road, Hongkou, Shanghai 200080, P.R. China

E-mail: du_lf@163.com

Key words: ultrasound, drug delivery, destruction, microbubble, nano-microcapsules

\section{Contents}

1. Introduction

2. Material selection and production of nano-microcapsules

3. Factors affecting the targeted delivery efficiency of nano-microcapsules

4. Research on overcoming the hindrances of nanoparticle delivery

5. Research progress on the delivery efficiency of drug-loaded nano-microcapsules

6. Possible mechanisms for the promotion of nano-microcapsule delivery

7. Research progress on nano-microcapsules delivery system mediated by UTMD

8. Conclusion

\section{Introduction}

An optimal drug delivery method is required to ensure safety and high efficiency of delivery. The nanoparticle has recently become one of the most popular and promising non-viral vectors (1) and has several advantages compared to viral vectors, such as lack of pathogenicity, lack of immunogenicity, biodegradability, wide range of host cells or tissues and diversification of loadings. The diameter of nanoparticles is $1 / 60-1 / 60,000$ that of a cell. Therefore, drug-loaded nano-microcapsules are able to pass through several insurmountable obstacles and ingress the interior of cells and tissues for targeted therapy $(1,2)$. However, drug delivery efficiency does not appear to reach satisfactory therapeutic levels, particularly under specific physiological or pathological conditions.

The oscillation and destruction of microbubbles, as well as microstreaming and radiation forces generated by ultrasound-targeted microbubble destruction (UTMD) may result in the rupture of stalwart barriers, such as the blood-brain barrier, dense connective tissue and the cell membrane structure, allowing more nano-microcapsules into cells and tissues. Recent studies demonstrated that UTMD has considerably 
improved the efficiency of the nano-microcapsules drug delivery system (3-7).

\section{Material selection and production of nano-microcapsules}

Material selection. The materials used for manufacturing nano-microcapsules are classified as non-biodegradable and biodegradable. Non-biodegradable materials are able to protect DNA and RNA from digestion by enzymes, however, they may result in severe cytotoxicity and tissue necrosis (8-10). Biodegradable materials are highly biocompatible and are able to be decomposed by hydrolytic enzymes in the body and absorbed, ultimately metabolize to carbon dioxide and water through the tricarboxylic acid cycle and are excreted by the lungs, kidneys and skin. Therefore, biodegradable materials are considered the optimal choice and are widely used (11). Poly(lactic-co-glycolic acid) (PLGA) copolymer $(12,13)$, one of the most commonly used biodegradable polyester materials, may be used in all types of drug-loaded nano-microcapsules embedding proteins (3), amino acids (3), genes (3), vaccines (9), antigens and growth factors (4). PLGA has been approved by the FDA for human medical use, and is non-toxic and harmless $(10,14,15)$. Its crystallinity, solubility and water absorption capacity are regulated by modifying the proportion of polylactic and polyglycolic acid to control the rate of degradation, in order to meet the needs of the release of different embedded drugs (16-19).

Production of nano-microcapsules. Nanoparticle-producing technologies are currently classified into three categories, the mechanical pulverization, physical dispersion and chemical synthesis methods. Different types of nanoparticles are manufactured by different techniques and processes. The mechanical smashing method is a technique during which the mass is broken into nanoparticles by a high-speed rotary mill, jet mill, ultrasound, ball mill or colloid mill. The solvent evaporation and emulsification/solvent diffusion methods (physical methods) are suitable for producing nanosuspensions. The chemical synthesis method uses the hydrophobic segments of polymers to synthesize surface-active block copolymers. Several studies on nanoparticles successfully loading DNA (7), siRNA (7), anticancer drugs such as cisplatin (20) and mitoxantrone (21), and antiparasitic drugs such as pentamidine (22) and albendazole (22) were recently published. The encapsulation efficiency of drugs is affected by factors such as material and emulsifier concentration and intensity of the ultrasonic irradiation and the release rate is regulated by the proportion of various components of the nanomaterial and the $\mathrm{pH}(20,23)$.

\section{Factors affecting the targeted delivery efficiency of nano-microcapsules}

Size of the nano-microcapsule. Nano-microcapsules may be used for the treatment of a variety of diseases, particularly tumors. Different sizes of nanoparticles are selective for different tumor tissues. In general, nano-microcapsules $\sim 150-300 \mathrm{~nm}$ readily accumulate in the liver and spleen and nano-microcapsules $\sim 30-150 \mathrm{~nm}$ are prone to accumulate in the bone marrow, heart and kidneys. Particularly small nano-microcapsules, with a diameter of $\sim 20-30 \mathrm{~nm}$ are usually cleared by the kidneys prior to ingressing the target tissues (24).

Electric charges borne on the surface of nano-microcapsules. The negative electric charges on the surface of nano-microcapsules limit their combination with certain gene drugs as well as with several target tissues and cells, particularly tumor cells $(25,23)$.

Monitoring of the immune system. Nano-microcapsules that enter the human body may be cleared away as foreign bodies by the mononuclear phagocytes of the reticuloendothelial system in the liver and spleen (24).

High expression of specific antigens or receptors on the surface of tumor cells. High expression of specific antigens or receptors on the surface of tumor cells or tumor vascular endothelial cells is moderately or not expressed on the suface of normal cells or normal vascular endothelial cells $(26,27)$.

\section{Research on overcoming the hindrances of nanoparticle delivery}

Prolonging the circulation time of nano-microcapsules. Modifying PLGA with monomethyl ether polyethylene glycol (mPEG) may shield some of the surface charges of the complex and evade clearance by the body's immune system, consequently prolonging the nano-microcapsules residence time in the systemic circulation $(28,29)$.

Increasing the rate of gene drug encapsulation by increasing the amount of surface positive charges to promote delivery efficiency. The positive charges on the surface of PLGA are distinctly increased following its combination with poly-L-lysine (PLL), which is able to generate electrostatic interactions with the negative charges carried by DNA/siRNA to improve the loading effect (27).

Active targeted delivery by targeting molecules modifying nano-microcapsules. Recently, several investigators reported that drug-loaded nano-microcapsules modified with specific target antibodies may actively recognize target tissues or target cells, increasing the efficiency of drug delivery $(26,27,30)$. The integrin $\alpha v \beta 3$ is a receptor that is highly expressed on the surface of a variety of tumor cells or malignant tumor vascular endothelial cells and not expressed or detected in normal tissue cells or mature vascular endothelial cells. mPEG-PLGA-PLL polymers modified with ligand analogs that contain the Arg-Gly-AsP sequence combine with $\alpha v \beta 3$ as antagonists to modify targeted delivery (29). Yoo et al (31) successfully constructed PEG-PLGA polymers modified with folic acid that encapsulated adriacin. Human oral squamous cell carcinoma cells exhibited increased uptake of nano-microcapsules modified with folic acid compared to unmodified ones in an in vitro study (32).

\section{Research progress on the delivery efficiency of drug-loaded nano-microcapsules}

Nano-microcapsule-targeted delivery technology has achieved some success; however, the gene transfection efficiency and 

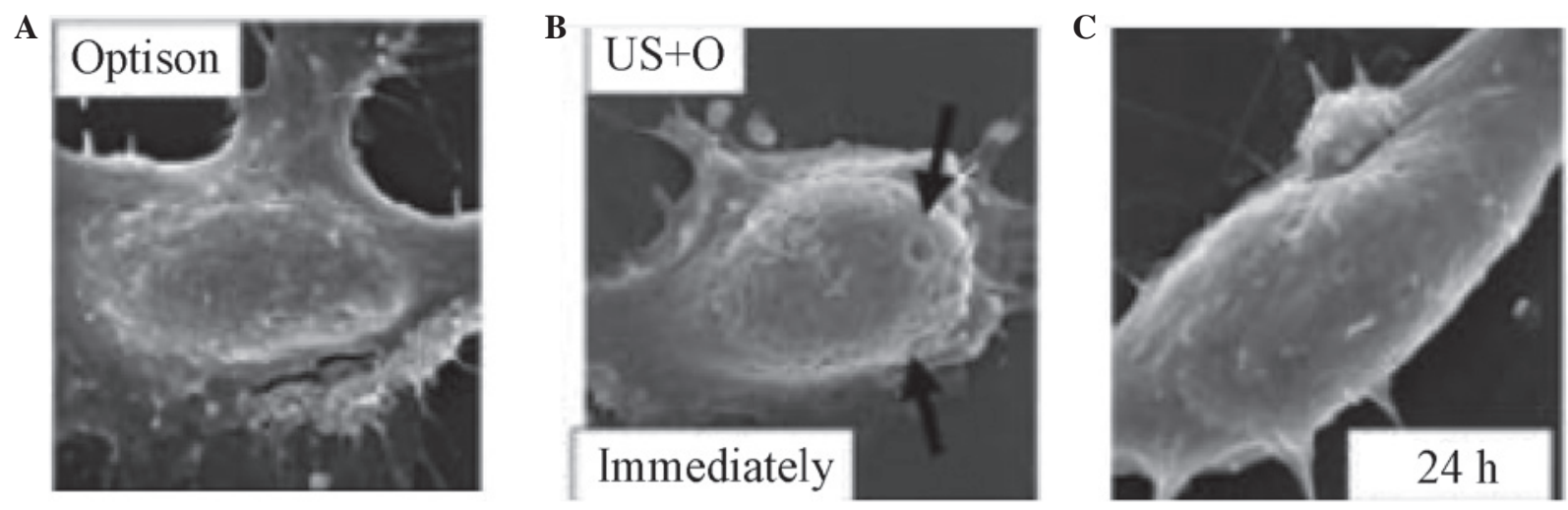

Figure 1. Formation of transient pores on the surfaces of cell membranes by ultrasonic irradiation under the electron microscope. (A) Pores in the cell membranes are not observed prior to ultrasonic irradiation. (B) Pores in the cell membranes are observed immediatedly following ultrasonic irradiation (arrows). (C) Pores in the cell membranes disappear $24 \mathrm{~h}$ after ultrasonic irradiation, van Wamel et al (37). US+O, ultrasound + Optison.

drug delivery efficiency remain low and do not satisfy the treatment demands.

A previous study conducted by de la Fuente et al (33) reported that plasmid DNA was delivered to the cornea and conjunctiva cells by a new type of nanocarrier synthesized by the bioadhesive polysaccharides hyaluronic acid and calcium silicate, the transfection efficiency of which was $15 \%$. Chen et al (20) suggested that the targeted therapy effect of nano-microcapsules containing mitoxantrone was slightly superior to intravenous chemotherapy in mouse breast cancer only to a certain extent. A previous study demonstrated that drug-loaded nano-microcapsules were extremely difficult to pass through the vitreous cavity, a grid-like barrier consisting of collagen fibers bridged by proteoglycans (34). However, the treatment of retinal diseases requires drugs to cross this barrier, which remains an intractable problem. Similarly, in pancreatic cancer (referred to as 'the king of cancer'), which exhibits a special pathological anatomy structure, drug-loaded nano-microcapsules faced significant resistance. A previous study (31) demonstrated that the peripancreatic tissue of normal pancreas as well as the leaf gap tissues that act as ingress and egress pathways to the blood, nerves and lymphatics of the normal pancreas are loose connective tissues. Furthermore, little leaf gap tissues of normal pancreas are connected to the retroperitoneal and peripancreatic loose connective tissues. By contrast, the tissues surrounding pancreatic cancer are dense connective tissues and the little leaf gaps of pancreatic cancer tissues are immersed in a substantial amount of fibrous tissue and lymphocytes. Therefore, it is difficult for nano-microcapsules to ingress pancreatic cancer tissues and identifying a way to promote nano-microcapsule delivery efficiency is of utmost importance. UTMD was recently verified to be a helpful tool to enhance nano-microcapsule delivery, for which possible mechanisms have been described.

\section{Possible mechanisms of UTMD for the promotion of nano-microcapsule delivery}

First, UTMD leads to the formation of transient openings on the surfaces of cell membranes through which nano-microcapsules are able to enter cells and deliver drugs and genes (35-38) (Fig. 1). Second, the greatly increased oxyradical generation

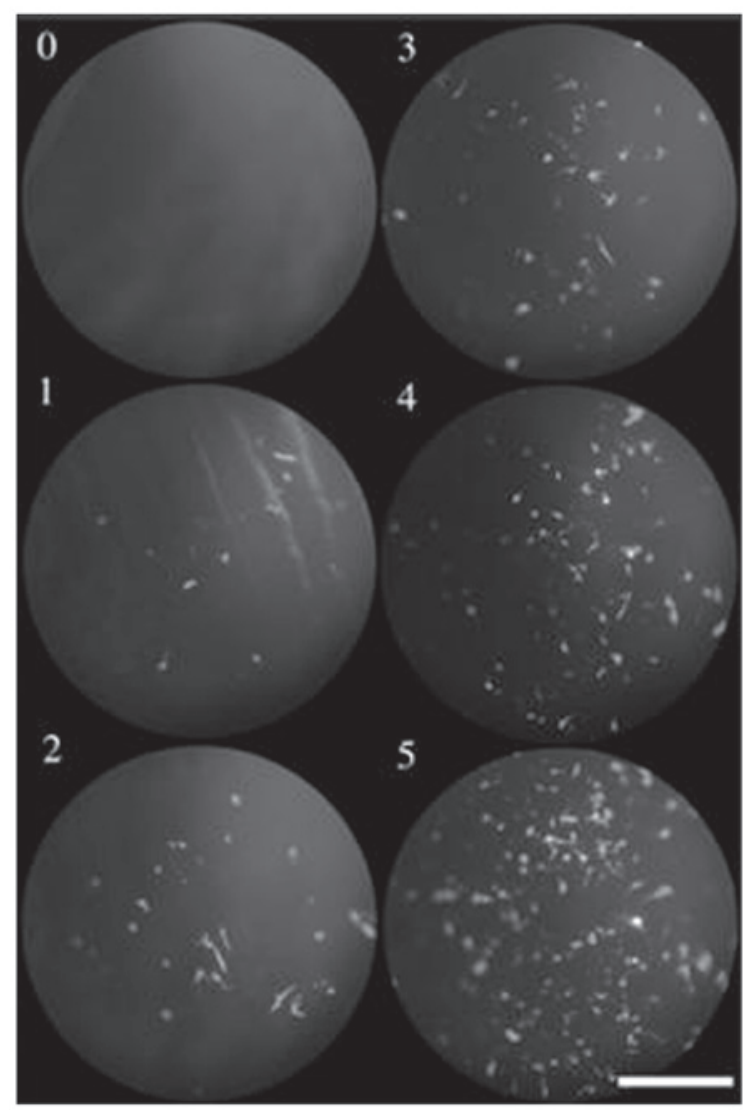

Figure 2. Representative photographs of scoring criteria. Gene transfer efficiency was expressed as a score of 0-5. Scoring was performed by three masked observers according to the following criteria: 0 , no positive cells; 1, 1-25 positive cells per field; $2,26-50$ positive cells per field; 3, 51-75 positive cells per field; $4,75-150$ positive cells per field; and 5, $\geq 151$ positive cells per field. Bar, $400 \mu \mathrm{m}$, Sonoda et al (41).

in cells under the effect of ultrasound (US) improves the permeability of cell membranes and promotes cellular uptake of nano-microcapsules (39). Third, US may increase endocytosis and activate cell membrane transport, thus enhancing the uptake of nano-microcapsules (40). US enables the local temperature of cell membrane to rise, which alters the liquidity of the membrane phospholipid bilayer and maximizes the cell membrane permeability. 
Although the mechanism underlying its action has not been fully elucidated, UTMD has played a significant role in mediating drug and/or gene delivery to several targets, such as eyes, tumors, skeletal muscle, heart and bone marrow stem cells $(3,4,34,40,41-46)$.

\section{Research progress on nano-microcapsules delivery system mediated by UTMD}

Eye. Sonoda et al (41) demonstrated that under the combination of US and Optison albumin-coated microbubbles, the green fluorescent protein (GFP) gene transfer to in vivo and in vitro rabbit corneal cells was greatly increased without apparent tissue damage, whereas US alone exerted a minimal enhancing effect on gene transfer (Fig. 2). Wu et al (42) also reported that using US in conjunction with commercially available SonoVue microbubbles safely enhanced GFP plasmid transfer to the mouse cornea in vivo. Another example of a successful gene transfer to the ocular surface mediated by UTMD was a study conducted by de la Fuente et al (33). By using a novel hyaluronic acid-Chitosan nanoparticle mediated by UTMD successful transfection of plasmid DNA in retinal pigment epithelium (RPE) cells in vitro and in vivo was achieved. Du et al (43) reported that UTMD is able to safely and effectively enhance siRNA-loaded nano-microcapsule delivery to RPE cells. Moreover, the most notable benefit of UTMD-mediated Cy3-siRNA loaded by nano-microcapsules was using the least amount of nano-microcapsules while maintaining a higher rate of uptake, which was achieved in rats in vivo and in vitro.

Tumor. Chumakova et al (44) reported that DNA-loaded nano-microcapsules produced from PLGA and PEI triggered by UTMD were successfully delivered to tumor cells in vivo. In addition, the gene transfection rate with UTMD was at least 8 times higher compared to that without UTMD. Hosseinkhani et al (45) demonstrated that cationic Dextran modified by PEG and US may target transfer plasmid DNA to fibrosarcoma cells efficiently. Rapoport et al (46) succeeded in synthesizing doxorubicin-containing polymer microcapsules and nano-microbubbles filled with gas, which were used for the treatment of mice bearing xenograft breast tumors, triggered by US. Doxorubicin was released from the polymer microcapsules to infiltrate target tumor interstitial tissues, leading to significant tumor shrinkage. Hauff et al (47) demonstrated that plasmid pU t651-MB packaged in inflatable nanoparticles combined with UTMD was effective in treating hepatocellular carcinoma in rats and gene expression in liver cancer cells was significantly increased. In the same manner, plasmid p16 as an anti-oncogene may effectively inhibit the growth of human pancreatic cancer cells. Yang et al (3) reported that gene-loaded Chitosan alginate particles combined with US significantly promoted the transfection efficacy of plasmid GFP in HeLa and 293T cells.

Heart and muscle. Bekeredjian et al (48) reported that luciferase reporter gene was target delivered to rat heart cells mediated by UTMD. After measuring the activity of luciferase and mRNA at different time points within 4 weeks, the investigators observed that the heart gene transfection efficiency mediated by UTMD was higher than that mediated by virus. Moreover, the transfection rate peaked after the first 4 days. Chappell et al (4) suggested that nano-microcapsules containing fibroblast growth factor 2 were largely deposited on the muscle tissue of rats mediated by UTMD.

\section{Conclusion}

Nano-microcapsule drug-loaded systems triggered by UTMD prolong the circulation time of the drug in the body and improve the drug concentration in target tissues, thus enhancing their efficacy. In addition, they reduce the frequency of drug administration. Therefore, they are regarded as fairly promising, particularly in cases with intractable malignant neoplasms. A previous study demonstrated that tumor cells were visualized through magnetic resonance concurrently with nano-microcapsule targeted therapy (49). Ke et al (50) of the Third People's Hospital affiliated with Peking University and Harbin Industry University, have synthesized a type of novel drug-loaded gold nano-microcapsule which may be useful for diagnosis and treatment. The gold nano-microcapsule combines the function of ultrasound contrast imaging with the function of photothermal therapy triggered by UTMD. Tumor position and size during the course of treatment is visualized and evaluated by enhanced ultrasound imaging of the polymer microcapsules. In addition, gold shells irradiated by laser generate high temperatures and destroy tumor tissues. However, the size of the gold nano-microcapsule is so minute that lesions may be visuaized only by using a great number of nanoparticles.

In conclusion, nano-microcapsules drug-loaded systems triggered by UTMD may play a critical role in therapy as well as imaging, which is a subject requiring further investigation.

\section{Acknowledgements}

The study was supported by grants from the National Natural Science Foundation of China (nos. 81171352 and 81271596).

\section{References}

1. Yang K, Feng L, Shi X and Liu Z: Nano-graphene in biomedicine: theranostic applications. Chem Soc Rev 10: 530-547, 2013.

2. Eldar-Boock A, Miller K, Sanchis J, et al: Integrin-assisted drug delivery of nano-scaled polymer therapeutics bearing paclitaxel. Biomaterials 32: 3862-3874, 2011.

3. Yang SJ, Chang SM, Tsai KC, et al: Effect of chitosan-alginate nanoparticles and ultrasound on the efficiency of gene transfection of human cancer cells. J Gene Med 12: 168-179, 2010.

4. Chappell JC, Song J, Burke CW, et al: Targeted delivery of nanoparticles bearing fibroblast growth factor-2 by ultrasonic microbubble destruction for therapeutic arteriogenesis. Small 4: 1769-1777, 2008

5. Park HJ, Lee J, Kim MJ, et al: Sonic hedgehog intradermal gene therapy using a biodegradable poly( $\beta$-amino esters) nanoparticle to enhance wound healing. Biomaterials 33: 9148-9156, 2012.

6. Kummitha CM, Malamas AS and Lu ZR: Albumin pre-coating enhances intracellular siRNA delivery of multifunctional amphiphile/siRNA nanoparticles. Int J Nanomedicine 7: 5205-5214, 2012.

7. Fields RJ, Cheng CJ, Quijano E, et al: Surface modified poly( $\beta$-amino ester)-containing nanoparticles for plasmid DNA delivery. J Control Release 164: 41-48, 2012.

8. Kumari A, Yadav SK and Yadav SC: Biodegradable polymeric nanoparticles based drug delivery systems. Colloids Surf B Biointerfaces 75: 1-18, 2010. 
9. Kang BC, Kang KS and Lee YS: Biocompatibility and long-term toxicity of lnnoPol implant a biodegradable polymer scaffold. Exp Anim 54: 37-52, 2005.

10. Itaka K, Ishii T, Hasegawa Y and Kataoka K: Biodegradable polyamino acid-based polycations as safe and effective gene carrier minimizing cumulative toxicity. Biomaterials 31 : 3707-3714, 2010.

11. Jere D, Jiang HL, Arote R, et al: Degradable polyethylenimines as DNA and small interfering RNA carriers. Expert Opin Drug Deliv 6: 827-834, 2009.

12. Nounou MI, Emmanouil K, Chung S, et al: Novel reducible linear L-lysine-modified copolymers as efficient nonviral vectors. J Control Release 143: 326-334, 2010.

13. Zhang Y, Chan HF and Leong KW: Advanced materials and processing for drug delivery: The past and the future. Adv Drug Deliv Rev 65: 104-120, 2012

14. Duan Y, Xu J, Lin Y, et al: A preliminary study on MeO-PEGPLGA-PEG-OMe nanoparticles as intravenous carriers. J Biomed Mater Res A 87: 515-523, 2008.

15. Brazeau GA, Sciame M, al-Suwayeh SA and Fattal E: Evaluation of PLGA microsphere size effect on myotoxicity using the isolated rodent skeletal muscle model. Pharm Dev Technol 1 : 279-283, 1996

16. Falamarzian A and Lavasanifar A: Optimization of the hydrophobic domain in poly(ethylene oxide)-poly(varepsiloncaprolactone) based nano-carriers for the solubilization and delivery of Amphotericin B. Colloids Surf B Biointerfaces 81: 313-320, 2010.

17. Gabler F, Frauenschuh S and Ringe J: Emulsion-based synthesis of PLGA-microspheres for the in vitro expansion of porcine chondrocytes. Biomol Eng 24: 515-520, 2007.

18. Falamarzian A and Lavasanifar A: Chemical modification of hydrophobic block in poly(ethylene oxide) poly(caprolactone) based nanocarriers: effect on the solubilization and hemolytic activity of amphotericin B. Macromol Biosci 10: 648-656, 2010.

19. Fan L, Li F, Zhang H, Wang Y, Cheng C, Li X, Gu CH, Yang Q, $\mathrm{Wu} \mathrm{H}$ and Zhang S: Co-delivery of PDTC and doxorubicin by multifunctional micellar nanoparticles to achieve active targeted drug delivery and overcome multidrug resistance. Biomaterials 31: 5634-5642, 2010

20. Chen M, Cooper HM, Zhou JZ, et al: Reduction in the size of layered double hydroxide nanoparticles enhances the efficiency of siRNA delivery. J Colloid Interface Sci 390: 275-281, 2013.

21. Du J, Shi QS, Sun Y, Liu PF, Zhu MJ, Du LF and Duan YR Enhanced delivery of monomethoxypoly(ethylene glycol)poly(lactic-co-glycolic acid)-poly l-lysine nanoparticles loading platelet-derived growth factor BB small interfering RNA by ultrasound and/or microbubbles to rat retinal pigment epithelium cells. J Gene Med 13: 312-323, 2011.

22. Duncan R: Polymer conjugates as anticancer nanomedicines. Nat Rev Cancer 6: 688-701, 2006.

23. Lee SH and Shin H: Matrices and scaffolds for delivery of bioactive molecules in bone and cartilage tissue engineering. Adv Drug Deliv Rev 59: 339-359, 2007.

24. Moghimi SM: Mechanisms of splenic clearance of blood cells and particles: towards development of new splenotropic agents. Adv Drug Deliv Rev 17: 103-115, 1995.

25. Nafee N, Taetz S, Schneider M, et al: Chitosan-coated PLGA nanoparticles for DNA/RNA delivery: effect of the formulation parameters on complexation and transfection of antisense oligonucleotides. Nanomedicine 3: 173-183, 2007.

26. Loomis K, Smith B, Feng Y, Garg H, Yavlovich A Campbell-Massa R, Dimitroy DS, Blumenthal R, Xiao X and Puri A: Specific targeting to B cells by lipid-based nanoparticles conjugated with a novel CD22-ScFv. Exp Mol Pathol 88: 238-249, 2010

27. Chen Y, Wu JJ and Huang L: Nanoparticles targeted with NGR motif deliver c-myc siRNA and doxorubicin for anticancer therapy. Mol Ther 18: 828-834, 2010.

28. Patil Y and Panyam J: Polymeric nanoparticles for siRNA delivery and gene silencing. Int J Pharm 367: 195-203, 2009.
29. Tahara K, Yamamoto H and Hirashima N: Chitosan-modified poly(D,L-lactide-co-glycolide) nanospheres for improving siRNA delivery and gene-silencing effects. Eur J Pharm Biopharm 74: 421-426, 2010.

30. Gunaseelan S, Gunaseelan K, Deshmukh M, et al: Surface modifications of nanocarriers for effective intracellular delivery of anti-HIV drugs. Adv Drug Deliv Rev 62: 518-531, 2010.

31. Yoo HS and Park TG: Folate receptor targeted biodegradable polymeric doxorubicin micelles. J Control Release 96: 273-283, 2004.

32. Yoo KS and Park TG: Folate receptor targeted biodegradable polymeric doxorubicin micelles. J Controlled Release 96 : 273-283, 2004

33. de la Fuente M, Seijo B and Alonso MJ: Novel hyaluronic acid-chitosan nanoparticles for ocular gene therapy. Invest Ophthalmol Vis Sci 49: 2016-2024, 2008.

34. Bishop P: The biochemical structure of mammalian vitreous. Eye (Lond) 10: 664-670, 1996.

35. Prentice P, Cushieri A, Dholakia K, Prausnitz M and Campbell P: Membrane disruption by optically controlled microbubble cavitation. Nat Phys 1: 107-110, 2005

36. Tachlibana K, Uchida T, Ogawa K, et al: Induction of cellmembrane porosity by ultrasound. Lancet 353: 1409, 1999.

37. van Wamel A, Kooiman K, Harteveld M, et al: Vibrating microbubbles poking individual cells: drug transfer into cells via sonoporation. J Control Release 112: 149-155, 2006.

38. Schlicher RK, Radhakrishna H, Tolentino TP, et al: Mechanism of intracellular delivery by acoustic cavitation. Ultrasound Med Biol 32: 915-924, 2006.

39. Juffermans LJ, Dijkmans PA, Musters RJ, et al: Transient permeabilization of cell membranes by ultrasound-exposed microbubbles is related to formation of hydrogen peroxide. Am J Physiol Heart Circ Physiol 291: H1595-H1601, 2006.

40. Miller DL and Gies RA: The interaction of ultrasonic heating and cavitation in vascular bioeffects on mouse intestine. Ultrasound Med Biol 24: 123-128, 1998.

41. Sonoda S, Tachibana K and Uchino E: Gene transfer to corneal epithelium and keratocytes mediated by ultrasound with microbubbles. Invest Ophthalmol Vis Sci 47: 558-564, 2006.

42. Wu Y, Du LF, Chen YD, et al: SonoVue and ultrasound-mediated pEGFP-N1 transfection to mouse cornea in an in vivo study. Chin J Ultrasonogr 17: 350-353, 2008.

43. Du J, Sun Y, Shi QS, Liu PF, Zhu MJ, Wang CH, Du LF and Duan YR: Biodegradable nanoparticles of mPEG-PLGA-PLL triblock copolymers as novel non-viral vectors for improving siRNA delivery and gene silencing. Int J Mol Sci 13: 516-533, 2012.

44. Chumakova OV, Liopo AV, Andreev VG, et al: Composition of PLGA and PEI/DNA nanoparticles improvesultrasound-mediated gene delivery in solid tumors in vivo. Cancer Lett 261: 215-225, 2008.

45. Hosseinkhani $\mathrm{H}$ and Tabata $\mathrm{Y}$ : Ultrasound enhances in vivo tumor expression of plasmid DNA by PEG-introduced cationized dextran. J Control Release 108: 540-556, 2005.

46. Rapoport N, Gao Z and Kennedy A: Multifunctional nanoparticles for combining ultrasonic tumor imaging and targeted chemotherapy. J Natl Cancer Inst 99: 1095-1106, 2007.

47. Hauff P, Seemann S, Reszka R, et al: Evaluation of gas-filled microparticles and sonoporation as gene delivery system: feasibility study in rodent tumor models. Radiology 236: $572-578$, 2005.

48. Bekeredjian R, Chen S, Frenkel PA, et al: Ultrasound-targeted microbubble destruction can repeatedly direct highly specific plasmid expression to the heart. Circulation 108: 1022-1026, 2003.

49. Torchilin VP: Passive and active drug targeting: drug delivery to tumors as an example. Handb Exp Pharmacol 197: 3-53, 2010.

50. Ke H, Wang J, Dai Z, et al: Gold-nanoshelled microcapsules: a theranostic agent for ultrasound contrast imaging and photothermal therapy. Angew Chem Int Ed Engl 50: 3017-3021, 2011. 